\title{
Impact of single-dose application of TGF- $\beta$, copper peptide, stanozolol and ascorbic acid in hydrogel on midline laparatomy wound healing in a diabetic mouse model
}

\author{
MORITZ A. KONERDING ${ }^{1}$, THOMAS ZIEBART ${ }^{1}$, TANJA WOLLOSCHECK ${ }^{1}$, \\ AXEL WELLMANN $^{2}$ and MAXIMILIAN ACKERMANN ${ }^{1}$ \\ ${ }^{1}$ Institute of Functional and Clinical Anatomy, University Medical Center of the Johannes Gutenberg \\ University Mainz, D-55126 Mainz; ${ }^{2}$ Institute of Pathology Celle, D-29222 Celle, Germany
}

Received February 23, 2012; Accepted April 12, 2012

DOI: $10.3892 / \mathrm{ijmm} .2012 .1005$

\begin{abstract}
Despite numerous advances and improvements in surgical techniques the incidence of incisional hernias after laparotomy remains high. The aim of this study was to investigate possible effects of single application of ascorbic acid, stanozolol, a synthetic anabolic steroid, copper peptide and transforming growth factor- $\beta$ (TGF- $\beta$ ) on laparotomy wound healing in an incisional wound model in diabetic mice. After diabetes induction with streptozotozin in Balb-c mice, midline laparatomies were carried out. Closure of the linea alba was followed by single-dose application of the agents dissolved in a hydrogel before skin closure. The functional outcome was assessed in terms of maximum tensile strength. In addition, vessel densities, collagen contents and proliferation, were measured. The breaking strength of the skin 14 days after surgery was significantly higher in ascorbic acid (AA)-treated incisional wounds, whereas the other agents did not show a significantly better functional outcome. No significant differences were seen in vessel densities. Collagen type III contents was higher in the AA-treated animals, whereas the percentage of Ki67-positive nuclei was lower compared to the other groups. These data underline the positive effect of topically applied ascorbic acid in wound healing.
\end{abstract}

\section{Introduction}

The incidence of incisional hernias after laparotomy because of visceral surgical interventions is in the range of 5-20\%

Correspondence to: Professor Moritz A. Konerding, Institute of Functional and Clinical Anatomy, University Medical Center of the Johannes Gutenberg University Mainz, Becherweg 13, D-55099 Mainz, Germany

E-mail: konerdin@uni-mainz.de

Key words: ascorbic acid, transforming growth factor- $\beta$, copper peptide, stanozolol, wound healing, diabetes mellitus, collagen synthesis, vessel density
$(1,2)$, frequently requiring surgical repair. The National Health Statistics Center quotes approximately 200,000 incisional hernia repairs each year in the United States alone (3).

A variety of mitogenic and/or angiogenic factors such as VEGF, FGF or PDGF have been successfully tested for experimental improvement of wound healing (4) and PDGF, -BB, bFGF and GM-CSF made it into the clinics. Novel delivery systems aiming at a continuous release of the factors or living cell therapy (4) as well as gene transfer strategies may further improve the therapeutic gains (5).

Transforming growth factor- $\beta$ (TGF- $\beta$ ) expression has been demonstrated throughout wound healing and has been shown to regulate many processes involved in tissue repair, including production of ECM, proteases, protease inhibitors, migration, chemotaxis and proliferation of macrophages, fibroblasts of the granulation tissue, epithelial and capillary endothelial cells (6). Copper plays a key role in angiogenesis and in the synthesis and stabilization of extracellular matrix skin proteins, which are critical processes of skin formation (7).

However, there is also reasonable evidence that easily available standard drugs or factors acting on or promoting the collagen synthesis, such as ascorbic acid (AA) or stanozolol, an anabolic steroid with fibrinolytic properties, are also beneficial for wound healing. Antioxidants such as Emblica officinalis were recently reported to improve wound healing through upregulation of collagen and extracellular signal-related kinases (8). Significant reductions of plasma AA concentrations in the post-operative period of patients are associated with an increase in post-operative complications (9). Pre-treatment with AA increased wound healing of excision wounds in mice exposed to whole body $\gamma$ irradiation (10).

This prompted us to compare the efficacy of topically applied AA in comparison to stanozolol, TGF- $\beta$, copper peptide and controls in midline laparotomy incisional wounds in a diabetic mouse model. The laparotomy model was chosen because of the high socio-economic impact of incisional hernias that complicate $11 \%$ of all abdominal wall closures (2). Maximum tensile strength testing was intended for measuring the functional outcome and histology and morphometry to elucidate possible modes of action. 


\section{Materials and methods}

Diabetes induction. Female BALB-C mice aged 4-5 months were injected intraperitoneally (i.p.) on Days $0,2,4$ and 7 with $150 \mathrm{mg} / \mathrm{kg}$ body weight (bw) streptozotocin (STZ; SigmaAldrich Chemie GmbH, Steinheim, Germany) solved in $0.9 \%$ $\mathrm{NaCl}$. Additional injections were administered if the blood glucose level was below $250 \mathrm{mg} / \mathrm{dl}$. Animals with a blood glucose level below $220 \mathrm{mg} / \mathrm{dl}$ received $150 \mathrm{mg} / \mathrm{kg}$ bw STZ, animals with blood glucose levels between $220-250 \mathrm{mg} / \mathrm{dl}$ received $120 \mathrm{mg} / \mathrm{kg}$ bw STZ until the desired blood glucose level of $250 \mathrm{mg} / \mathrm{dl}$ was reached. All mice were kept on a light/ dark $\left(12 \mathrm{~h} / 12 \mathrm{~h}\right.$ ) cycle at $23^{\circ} \mathrm{C}$ and received food (standard lab chow) and water ad libitum. The care of the animals was consistent with the guidelines laid down by law. All experiments were conducted with the approval of the local institutional review board.

Laparotomy incision wound. Surgery was performed under aseptic conditions. Mice were anesthetized intraperitoneally with $1 \mathrm{ml} / 100 \mathrm{~g}$ body weight Avertin (2,2,2-Tribromoethanol and Pentanol in PBS; Sigma-Aldrich, München, Germany). The skin of the ventral abdominal wall was shaved and wiped off with $70 \%$ isopropyl alcohol. After midline skin incision over a length of $18 \mathrm{~mm}$, the fascia was exposed and the linea alba was incised over a length of $15 \mathrm{~mm}$. The linea alba was closed again with 3-4 single button sutures Prolene ${ }^{\circledR}$ 6-0 (Ethicon; Johnson \& Johnson Medical GmbH, Norderstedt, Germany). After preparation of a subcutaneous pouch for the drugs solved in $0.2 \mathrm{ml}$ hydrogel, the skin was closed with 5-6 single button sutures.

Treatment groups and follow-up. Control animals (group 1) received hydrogel only, consisting of $12.5 \%$ hydroxypropylcellulose in phosphate buffer ( $\mathrm{pH}$ 7.4). Group 2 animals received hydrogel with $10 \mathrm{ng} / \mathrm{ml}$ TGF- $\beta$. Group 3 animals received hydrogel enriched with $100 \mu \mathrm{m}$ copper peptide $/ 100 \mathrm{~g}$, whereas group 4 animals received hydrogel added with $100 \mu \mathrm{m} / 100 \mathrm{~g}$ stanozolol. Group 5 animals received hydrogel with $100 \mu \mathrm{m} / 100 \mathrm{ml}$ AA. All gels were produced and portioned in a standardized procedure (Itemp, Aachen, Germany). The groups consisted of 12-14 animals each.

All animals survived the operation. Half of the animals were sacrificed on Day 3 and the other half 14 days after surgery. The skin sutures were removed after 6 days. After euthanasia with an overdose of pentobarbital (Sigma-Aldrich Chemie $\mathrm{GmbH}$ ) the tissue was sampled for biomechanical and histologic evaluation.

Tensile strength measurements. From all specimens obtained 14 days after surgery, $3-\mathrm{mm}$ wide test strips were punched out from the central area of the skin wound vertically to the craniocaudal axis. The test strips had a defined hour barbell form with 3-mm width at the narrowest part constituting a predetermined breaking point. The skin and subcutis were dissected from the linea alba and tested separately. The breaking strength test device consisted of 2 opposing gripping jaws to fix the tissue strip (11). The electric motor driven gripping jaws were moved apart with a constant strain rate of $0.5 \mathrm{~mm} / \mathrm{s}$ under displacement control. Time, force and displacement were recorded during the stretching of the tissue. Endpoint was the disrupture of the sample. A position encoder (WA300) was used to register the stretching distance; a force transducer (S2, maximum value $150 \mathrm{~N}$ ) was used to quantify the power impacting on the tissue strip. The resulting values were processed by a multiple channel PC measuring device (Spider 8) and plotted as way-power curve (software: Catman 4.5; all HBM Hottinger Baldwin Messtechnik, Darmstadt, Germany). The maximum breaking strength was determined from the stress-strain curve. Specimens sampled on Day 3 after surgery were not considered for biomechanical testing due to the early time point.

Histology and immunohistochemistry. Ten-micrometer thick slides of paraffin embedded probes were stained with hematoxylin and eosin (H\&E) according to standard protocols. H\&E sections were used to assess the morphology of the scars and the thicknesses as measure for the progress of remodeling. The thickness of the individual skin layers were measured with the image analyzing software Diskus 4.80 (Hilgers Königswinter, Germany). The sections were quantified in a blinded manner by an independent observer. Picrosirius red staining was used to quantify the collagen content by means of polarization microscopy. Collagen content was expressed as percentage of red-stained pixels assessed with the image analyzing software KS300 (Kontron AG, Eching, Germany).

Immunohistochemical staining of endothelial cells was performed using a monoclonal antibody against CD31 (BD Biosciences Pharmingen, Heidelberg, Germany). Antibody binding was visualized via a 3 -step staining procedure using a biotinylated polyclonal anti-rat Ig-G secondary antibody (DakoCytomation GmbH, Hamburg, Germany) and the streptavidin horseradish peroxydase reaction together with the DAB detection system. The vessel densities were assessed using a Weibel grid and expressed as percentual vessel surface area. The proliferation rate was assessed using a Weibel grid to count anti-Ki67-positive cells in defined areas.

Statistical analysis. The unpaired Student's t-test for samples of unequal variances was used to calculate statistical significance. The data were expressed as the mean $\pm \mathrm{SD}$. The significance level for the sample distribution was defined as $\mathrm{p}<0.05$.

\section{Results}

Macroscopic appearance. As shown in Fig. 1, the wounds of all animals were correctly adapted on Day 3 showing on the peritoneal side hyperemia and diffuse minor hemorrhages that were evident also on Day 14. The main branches of the inferior epigastric arteries appeared dilated. No group differences were observed.

Tensile strength. Fig. 2 shows the lowest breaking strength in the controls with a mean of $0.38 \mathrm{~N}$. The highest maximum tensile strengths were found in the AA group with a mean of $0.74 \mathrm{~N}$. The stanozolol group showed a mean of $0.56 \mathrm{~N}$. There was a significant difference of the AA group in comparison to the control group $(\mathrm{p}=0.002)$ and to the TGF- $\beta$ group $(\mathrm{p}=0.04)$. The variability of the values of the AA group was significantly less than in all other groups. The other groups failed to reach 


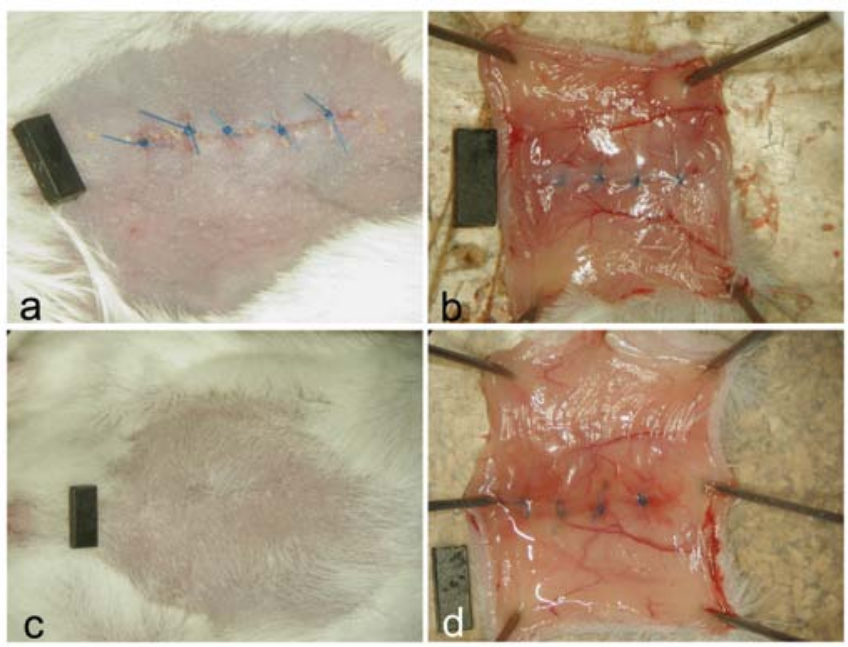

Figure 1. (a) Laparotomy suture 3 days after surgery and treatment with ascorbic acid (AA). (b) shows the abdominal wall of the same specimen from the peritoneal site. (c and d) Laparotomy 14 days after surgery and stanozolol treatment. Note the hyperemia and the dilated vessels from the peritoneal aspect.

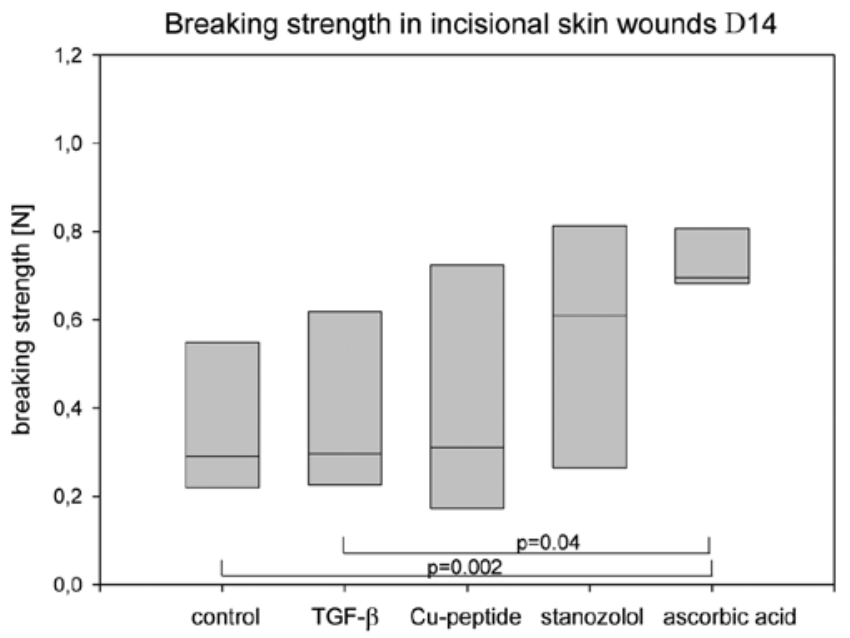

Figure 2. Maximum tensile strengths of the laparotomy skin 2 weeks after surgery. Box plots with $25^{\text {th }}, 50^{\text {th }}, 75^{\text {th }}$ percentile.

the level of significance as compared to the control group because of the high dispersion coefficient.

The maximum tensile strengths of the linea alba itself did not show significant differences between the individual groups (data not shown). Histology, however, revealed that this result was mainly caused by the small width of the linea alba $(150-300 \mu \mathrm{m})$ which resulted in test specimens that consisted mainly of musculature. In all cases the breaking did not occur within the scar, but paramedially in the musculature. Thus, a reliable measurement of the impact of the agents on the healing of the linea alba scar itself was not possible.

Collagen contents. The percentage of collagen type I and III in the area of interest was assessed in picrosirius red stained specimens (Fig. 3). Type III collagen was significantly increased in the skin of the stanozolol and AA groups compared to the control group on Day 14 (Fig. 4a). The collagen type I contents,
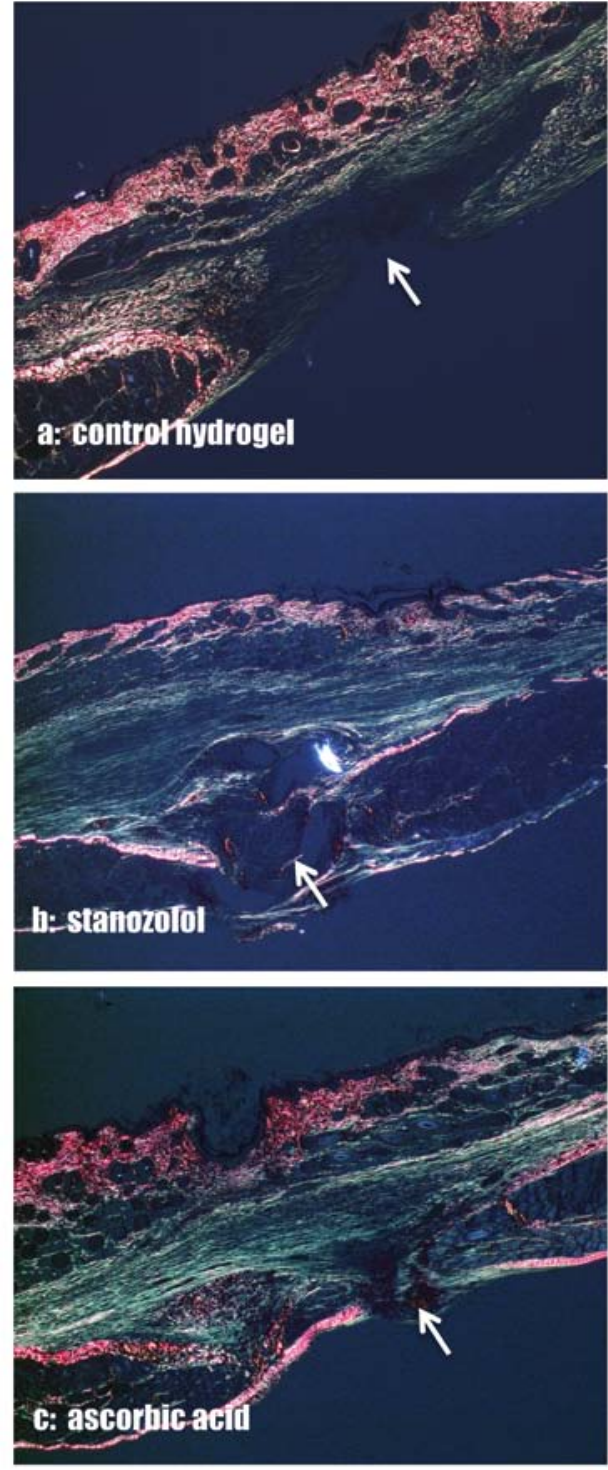

Figure 3. Collagen type III depiction in picro-sirius red stained specimens in polarisation microscopy. The collagen type III fibers appear in green. Arrows mark the midline linea alba. The peritoneum is orientated in all specimens to the bottom, the epidermis to the top. (a) control, (b) stanozolol and (c) ascorbic acid treatment. Magnification x100.

however, did not vary between the groups (Fig. 4b). In the linea alba itself no differences in the collagen type I/III ratios were observed.

Thicknesses of the scar tissue. Measurements of the scar dimensions in the midline show that significantly higher values were observed in copper peptide and stanozolol animals than in the other groups (Fig. 5). Despite the higher values for collagen type III, AA-treated wounds did not result in higher scar thicknesses.

Proliferation rate. Anti-Ki67 stains revealed no significant differences in the proliferation status of the linea alba scar (Fig. 6a), but significantly lower values for AA-treated animals in the skin layer (Fig. 6b), indicating an earlier remodelling process. 

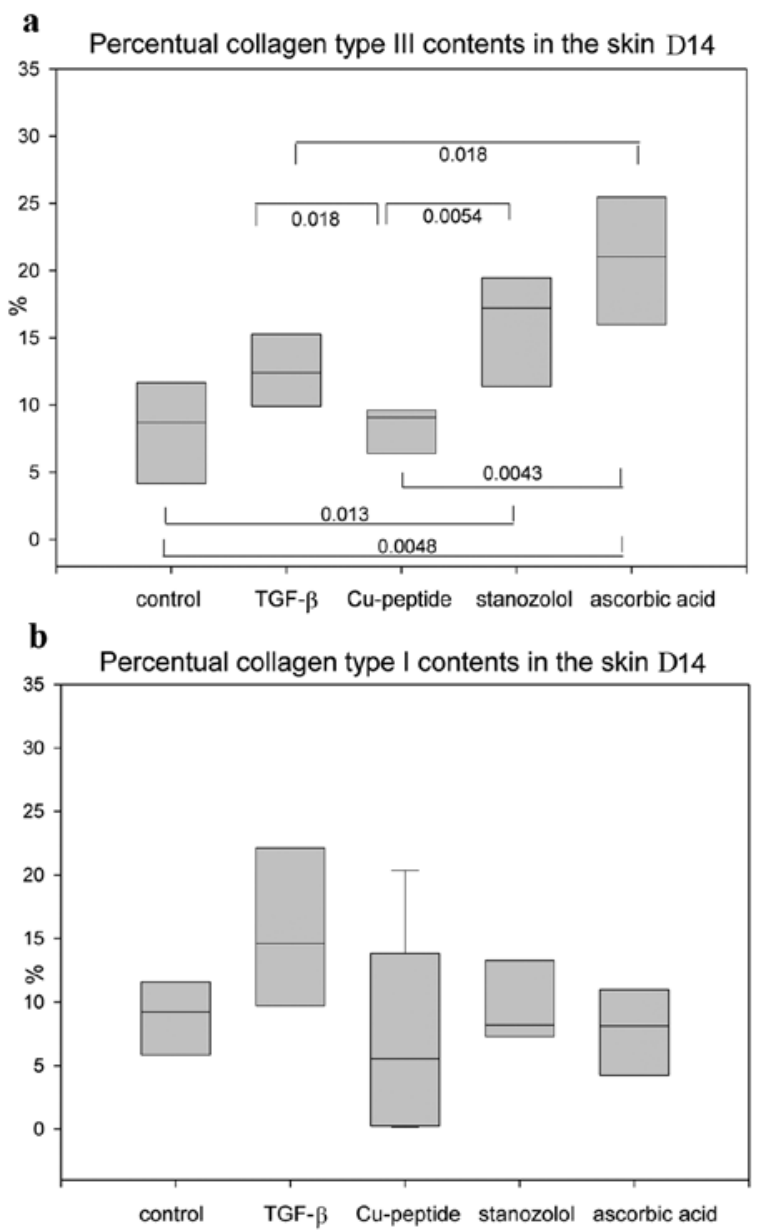

Figure 4. (a) Percentual collagen type III and (b) type I contents in the skin 14 days after surgery. Box plots indicating $25^{\text {th }}, 50^{\text {th }}$ and $75^{\text {th }}$ percentile.

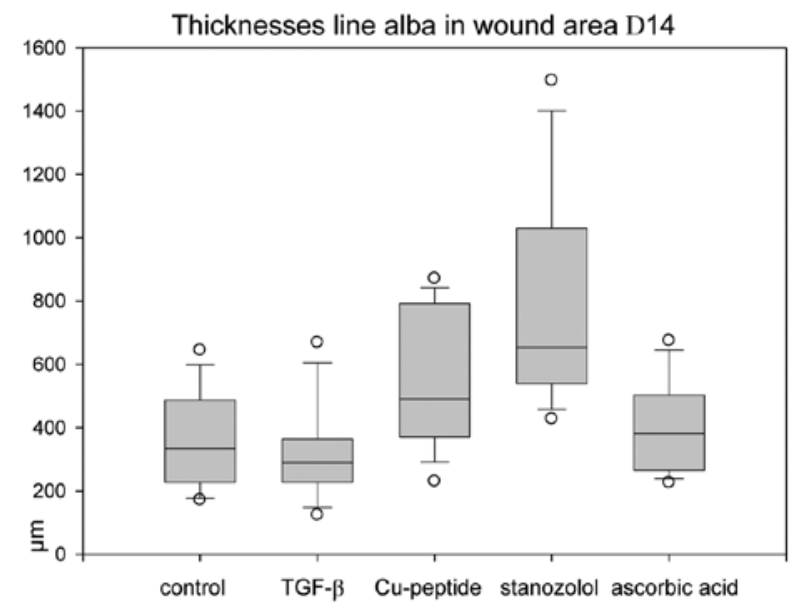

Figure 5. Scar tissue thicknesses 14 days after midline laparotomy. Box plots indicating $5^{\text {th }}, 10^{\text {th }}, 25^{\text {th }}, 50^{\text {th }}, 75^{\text {th }}, 90^{\text {th }}$ and $95^{\text {th }}$ percentile.

\section{Discussion}

Irrespective of the identification of numerous risk factors, such as suboptimal closure techniques or wound infections (12-16), the incidence of incisional hernias after laparotomy remains a Percentage of Ki67-positive nuclei in the linea alba D14

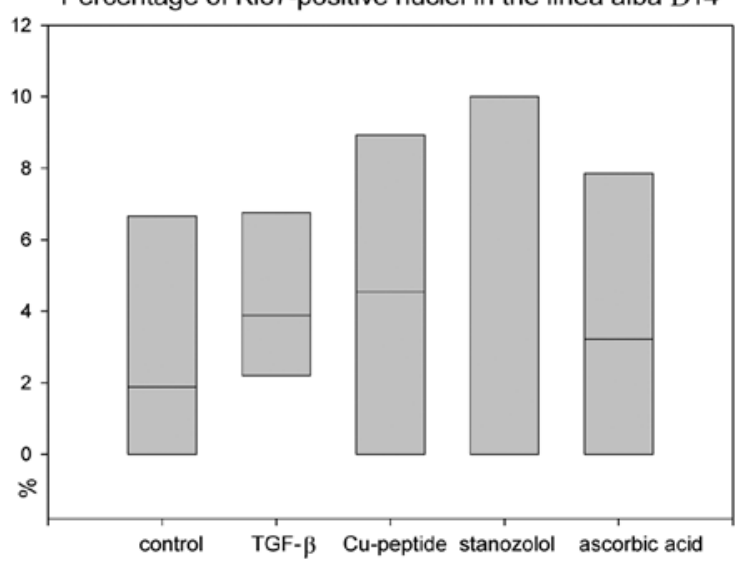

b Percentage of Ki67-positive nuclei in skin layer D14

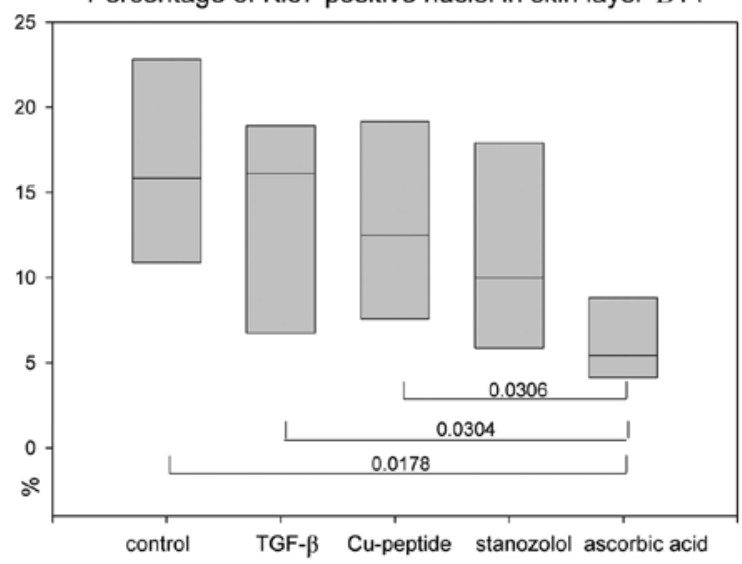

Figure 6. (a) Percentage of Ki67-positive nuclei in the linea alba and in the skin layers 14 days after surgery. Box plots indicating $25^{\text {th }}, 50^{\text {th }}$ and $75^{\text {th }}$ percentile.

high. Midline laparatomies may result in incidences of $15-20 \%$ (1). This calls for new approaches in optimised wound treatment, e.g., by application of pro-angiogenic and pro-mitogenic substances or agents. In a rat model of incisional hernias, significant increases of fascial wound breaking strength were seen after treatment with delayed release of bFGF (17). The aim of our study was to elucidate possible effects of single doses of AA and stanozolol in comparison to TGF- $\beta$ and copper peptide on laparotomy wound healing.

Wound healing is a complex process with numerous cell types, signal transduction pathways and factors involved. This complex process may be disturbed by diseases like diabetes, which is known to be associated with a variety of alterations in connective tissue metabolism, as a result of which diabetics face the problem of poor wound healing (18). Diabetic macroangiopathy and microangiopathy make tissues relatively ischemic, impairing wound healing and increasing the risk of infection (19). Macrophages are dysfunctional due to glycosylation and thus they do not adequately debride wounds or combat infection $(19,20)$. Angiogenesis may be severely impaired, further delaying wound healing (19). This results, even in induced murine diabetes, in more pronounced effects of proangiogenic and promitogenic growth factors in diabetic than in normoglycemic animals $(21,22)$. 
Stanozolol was developed in the 1950 s in an attempt to dissociate the anabolic and androgenic effects of testosterone. The anabolic steroid stanozolol has been particularly helpful because it has one of the largest anabolic/androgenic ratios. In addition, stanozolol has substantial fibrinolytic properties. Stanozolol is approved for use in the treatment of hereditary angioedema. Stanozolol was also significantly capable of stimulating the pro-collagenase production by skin fibroblasts (23). It was also reported that stanozolol stimulates the production of prostaglandin E2 (PGE2) and the matrix metalloproteinase collagenase and stromelysin in human skin fibroblasts (24). Falanga et al (25) showed that stanozolol enhances collagen synthesis in vitro in a dose-dependent manner. Stanozolol also increases the mRNA levels of alpha1 (I) and alpha1 (III) procollagen and, to a similar extent, upregulated TGF- $\beta 1$ mRNA and peptide levels.

AA contributes to several metabolic processes including efficient hydroxylation of HP in elastin, collagen and proteins with collagenous domains. AA is required for the hydroxylation of proline residues in procollagen and HP stabilizes the collagen triple helical structure. Consequently, ascorbate stimulates procollagen secretion (26). Low levels of vitamin C may result in higher incidence of complications and retarded wound healing $(4,27)$.

Copper introduced into wound dressings was hypothesized to enhance wound repair (7). Application of wound dressings containing copper oxide to wounds inflicted in genetically engineered diabetic mice (C57BL/KsOlaHsd-Lepr ${ }^{\mathrm{db}}$ ) resulted in increased gene and in situ upregulation of proangiogenic factors, increased blood vessel formation and enhanced wound closure as compared to control dressings (7).

Among many molecules known to influence wound healing, TGF- $\beta 1$ has the broadest spectrum of actions, affecting all cell types that are involved in all stages of wound healing (26). Both positive and negative effects of TGF- $\beta 1$ on wound healing have been reported (28).

Our study showed that single doses of AA only had a significant effect on the healing of incisional skin wounds in terms of higher tensile strength. TGF- $\beta$ and copper peptide had no effect, whereas stanozolol treated wounds had a tendency towards higher values. The content of Collagen type III was also markedly increased in animals treated with AA or stanozolol. The proliferation marker Ki67 showed the lowest values for AA-treated incisional wounds indicating a faster healing and remodelling in the skin layer, but not in the linea alba connective tissue.

The limited beneficial results of our experimental setting indicate that single dose treatment is inferior to a repetitive or delayed release of the factors. Stumpf et al (29) developed a drug-eluting platform device that enables continuous release of AA from a wound dressing. It would be desirable to develop release systems that may be implanted at time of surgery in order to optimize or accelerate laparotomy wound healing.

\section{Acknowledgements}

The authors appreciate the technical assistance of Kerstin Bahr, Institute of Functional and Clinical Anatomy, University Medical Center of the Johannes Gutenberg University Mainz.

\section{References}

1. Höer J, Anurov M, Titkova S, Klinge U, Töns C, Ottinger A and Schumpelik V: Influence of suture material and suture technique on collagen fibril diameters in midline laparotomies. Eur Surg Res 32: 359-367, 2000.

2. Santora TA and Roslyn JJ: Incisional hernia. Surg Clin North Am 73: 557-570, 1993.

3. Graves EJ and Giullum BS: Detailed diagnoses and procedures, National hospital discharge survey, 1995. Vital Health Stat 13: 1-146, 1997.

4. Langemo D, Anderson J, Hanson D, Hunter S, Thompson P and Posthauer ME: Nutritional considerations in wound care. Adv Skin Wound Care 19: 297-303, 2006.

5. Giunta RE, Holzbach T, Taskov C, Holm PS, Konerding MA, Schams D, Biemer E and Gänsbacher B: AdVEGF165 gene transfer increases survival in overdimensioned skin flaps. J Gene Med 7: 297-306, 2005.

6. Valluru M, Staton CA, Reed MW and Brown NJ: Transforming growth factor beta and endoglin signaling orchestrate wound healing. Front Physiol 2: 89-99, 2011.

7. Borkow G, Gabbay J, Dardik R, Eidelman AI, Lavie Y, Grunfeld Y, Ikher S, Huszar M, Zatcoff RC and Marikovsky M: Molecular mechanisms of enhanced wound healing by copper oxide-impregnated dressings. Wound Repair Regen 18: 266-275, 2010.

8. Sumitra M, Manikandan P, Gayathri VS, Mahendran P and Suguna L: Emblica officinalis exerts wound healing action through upregulation of collagen and extracellular signal-regulated kinases (ERK1/2). Wound Repair Regen 17: 99-107, 2009.

9. Rümelin A, Humbert T, Luhker O, Drescher A and Fauth U: Metabolic clearance of the antioxidant ascorbic acid in surgical patients. J Surg Res 129: 46-51, 2005.

10. Jagetia GC, Rajanikant GK and Mallikarjun Rao KV: Ascorbic acid increases healing of excision wounds of mice whole body exposed to different doses of gamma-radiation. Burns 33: 484-494, 2007.

11. Wolloscheck T, Gaumann A, Terzic A, Heintz A, Junginger T, and Konerding MA: Inguinal hernia: measurement of the biomechanics of the lower abdominal wall and the inguinal canal. Hernia 8: 233-241, 2004.

12. Israelsson LA and Jonsson T: Suture length to wound length ratio and healing of midline laparotomy incisions. Br J Surg 80: 1284-1286, 1993.

13. Hoer JJ, Junge K, Schachtrupp A, Klinge U and Schumpelick V: Influence of laparotomy closure technique on collagen synthesis in the incisional region. Hernia 6: 93-98, 2002.

14. Wissing J, van Vroonhoven TJ, Schattenkerk ME, Veen HF, Ponsen RJ and Jeekel J: Fascia closure after midline laparotomy: results of a randomized trial. Br J Surg 74: 738-741, 1987.

15. Gutiérrez de la Pena C, Medina Achirica C, Dominguez-Adame E and Medina Diéz J: Primary closure of laparotomies with high risk of incisional hernia using prosthetic material: analysis of usefulness. Hernia 7: 134-136, 2003.

16. de Vries Reilingh TS, van Geldere D, Langenhorst B, de Jong D, van der Wilt GJ, van Goor H and Bleichrodt RP: Repair of large midline incisional hernias with polypropylene mesh: comparison of three operative techniques. Hernia 8: 56-99, 2004.

17. Dubay DA, Wang X, Kuhn MA, Robson MC and Franz MG: The prevention of incisional hernia formation using a delayedrelease polymer of basic fibroblast growth factor. Ann Surg 240: 179-186, 2004.

18. Wilkinson-Berka JL and Fletcher EL: Angiotensin and bradykinin: targets for the treatment of vascular and neuro-glial pathology in diabetic retinopathy. Curr Pharm Des 10: 33133330, 2004.

19. Falanga V: Wound healing and its impairment in the diabetic foot. Lancet 366: 1736-1743, 2005.

20. Aronson D and Rayfield EJ: How hyperglycemia promotes atherosclerosis: Molecular mechanisms. Cardiovasc Diabetol 1: $1-10,2002$.

21. Ackermann M, Wolloscheck T, Wellmann A, Li VW, Li WW and Konerding MA: Priming with a combination of proangiogenic growth factors improves wound healing in normoglycemic mice. Int J Mol Med 27: 647-653, 2011.

22. Ackermann M, Wolloscheck T, Wellmann A, Li VW, Li WW and Konerding MA: Priming with a combination of proangiogenic growth factors enhances wound healing in streptozotocininduced diabetes in mice. Eur Surg Res 47: 81-89, 2011. 
23. Wright JK, Smith AJ, Cawston TE and Hazleman BL: The effect of the anabolic steroid, stanozolol, on the production of procollagenase by human synovial and skin fibroblasts in vitro. Agents Actions 28: 279-282, 1989.

24. Ellis AJ, Cawston TE and Mackie EJ: The differential effects of stanozolol on human skin and synovial fibroblasts in vitro: DNA synthesis and receptor binding. Agents Actions 41: 37-43, 1994.

25. Falanga V, Greenberg AS, Zhou L, Ochoa SM, Roberts AB, Falabella A and Yamaguchi Y: Stimulation of collagen synthesis by the anabolic steroid stanozolol. J Invest Dermatol 111: 1193-1197, 1998.

26. Kaplan B, Gonul B, Dincer S, Dincer Kaya FN and Babul A: Relationships between tensile strength, ascorbic acid, hydroxyproline, and zinc levels of rabbit full-thickness incision wound healing. Surg Today 2004: 747-751, 2004.
27. Desneves KJ, Todorovic BE, Cassar A and Crowe TC: Treatment with supplementary arginine, ascorbic acid and zinc in patients with pressure ulcers: a randomised controlled trial. Clin Nutr 24: 979-987, 2005.

28. Wang XJ, Han G, Owens P, Siddiqui Y and Li AG: Role of TGF beta-mediated inflammation in cutaneous wound healing. $J$ Investig Dermatol Symp Proc 11: 112-117, 2006.

29. Stumpf U, Michaelis M, Klassert D, Cinatl J, Altrichter J, Windolf J, Hergenröther J and Scholz M: Selection of proangiogenic ascorbate derivatives and their exploitation in a novel drug-releasing system for wound healing. Wound Repair Regen 19: 597-607, 2011. 